\section{ORIGINAL RESEARCH}

\author{
E. Gifford \\ D. Drazin \\ J.C. Dalfino \\ A.K. Nair \\ J. Yamamoto \\ A.S. Boulos
}

\title{
The Effectiveness of Microballoon Angioplasty in Treating Middle Cerebral Artery Occlusion Beyond the Bifurcation
}

\begin{abstract}
BACKGROUND AND PURPOSE: PTA effectively treats vasospasm and arterial disease in peripheral, coronary, and large proximal cerebral vessels but rarely reaches small vessels like the distal MCA beyond the bifurcation. Our purpose was to evaluate the safety and efficacy of PTA for distal MCA occlusions in acute ischemic stroke.
\end{abstract}

MATERIALS AND METHODS: Seven patients had strokes in branches of their MCAs. Following diagnostic angiography, all received microballoon angioplasty and various combinations of IA alteplase (rtPA), abciximab, and/or nitroglycerin. Two also underwent stent placement. Comprehensive retrospective review of the patients' records was performed. Patients' NIHSS scores were reassessed before discharge. Recanalization was evaluated by angiography after treatment and at follow-up.

RESULTS: PTA was successfully performed in 7 patients without treatment-associated intracerebral hemorrhage. Two patients received distal MCA angioplasty as a secondary intervention: 1 following failed treatment with a Merci retriever and the other after successful removal of proximal clot with a Merci retriever. One patient did not recover from the initial ischemic event despite an excellent angiographic result. Complete recanalization (modified TIMI grade 4) was achieved in 4 patients and near-complete recanalization with mild flow deficit (modified TIMI grade 3), in 3 patients.

CoNCLUSIONS: PTA of the distal MCA with a microballoon is safe and effective for acute ischemic stroke. This case series demonstrates that endovascular treatment beyond the MCA bifurcation can dramatically reverse neurologic deficits.

\begin{abstract}
ABBREVIATIONS: $\mathrm{AMC}=$ Albany Medical Center; $\mathrm{AP}=$ anteroposterior; $\mathrm{CTA}=\mathrm{CT}$ angiography; $\mathrm{ED}=$ emergency department; $\mathrm{HH}=$ Hunt and Hess; IA = intra-arterial; ICA = internal carotid artery; Intra = drugs delivered between treatments; IV = intravenous; $L=$ left; MCA = middle cerebral artery; $\mathrm{mRS}=$ modified Rankin Score; NIHSS = National Institutes of Health Stroke Scale; Post $=$ drugs delivered following mechanical treatment; Pre = spasmolytic agents delivered prior to mechanical treatment; PTA = percutaneous transluminal angioplasty; $R=$ right; $R H F=$ righthanded female; rtPA = recombinant tissue plasminogen activator; TIMI = Thrombolysis in Myocardial Infarction
\end{abstract}

D uring the past decade, modifications in treatment and advancements in medical-device therapy have worked to extend the time available for treatment of acute ischemic stroke. The traditional 3-hour window for administration of IV alteplase (Activase) (rtPA) has been modified to include a 6-hour window for IA chemical thrombolysis with angiographic guidance to the site of occlusion. ${ }^{1,2}$ New mechanical devices for clot retrieval have sought to expand that time window to 8 hours. ${ }^{3}$

While IA chemical thrombolysis allows concomitant device treatment at the site of occlusion, this is less commonly attempted at distal vessel sites due to the increased risk of device perforation and intracranial hemorrhage. Distal branches of the MCA exhibit increased vessel tortuosity and smaller caliber, which previously made them unlikely candi-

Received July 29, 2009; accepted after revision February 23, 2010.

From the Division of Neurosurgery (E.G., J.C.D., A.K.N., J.Y., A.S.B.), Albany Medical Center, Albany, New York; and Department of Neurosurgery (D.D.), Cedars-Sinai Medical Center, Los Angeles, California.

Paper previously presented at: Annual Meeting of the Congress of Neurological Surgeons, September 20-25, 2008; Orlando, Florida.

Edward Gifford and Doniel Drazin are co-first authors.

Please address correspondence to Alan S. Boulos, MD, FACS, Division of Neurosurgery, Albany Medical Center, 47 New Scotland Ave, Albany, NY 12208; e-mail: alan.boulos @gmail.com

DOI 10.3174/ajnr.A2099 dates for mechanical intervention. Documented options for mechanical treatment of proximal MCA branches have included stent placement, clot retrieval, snare retrieval, and PTA. ${ }^{3-8}$ Although there have been attempts to achieve vessel recanalization in the M2 segment of the MCA by using device therapy, these have been limited to the placement of selfexpanding stents and deflated balloons over guidewires. ${ }^{5,7}$

These limitations are of particular significance because though M2 and M3 branches are smaller vessels of the cerebral vasculature, there is a significant risk that lesions at these locations will produce significant neurological disability. In addition, when treated with IV rtPA, these types of infarctions do have a propensity to develop hemorrhagic conversions. ${ }^{9}$

To date, we have not found any literature examining the safety and efficacy of PTA with concomitant IA thrombolysis for the treatment of acute ischemic stroke in the M2 or M3 division of the MCA. Our goal was to examine angiographic and clinical outcomes as well as complications of PTA to the M2 or M3 segments for patients presenting with acute ischemic stroke.

\section{Materials and Methods}

\section{Patient Demographics}

Seven patients were treated with PTA between September 2005 and December 2007. The mean patient age was 67 years (range, 28- 


\begin{tabular}{|c|c|c|c|c|c|}
\hline $\begin{array}{l}\text { Case } \\
\text { No. } \\
\end{array}$ & Occlusion Sites & Spasmolytic Agents & $\begin{array}{c}\text { Timing of } \\
\text { Drug Therapy }\end{array}$ & Mechanical Device & $\begin{array}{l}\text { No. of Balloon } \\
\text { Inflations }\end{array}$ \\
\hline 1 & L M2 & ReoPro & Post & $\begin{array}{l}\text { Merci } \\
\text { Maverick, } 2 \times 9 \mathrm{~mm} \text {, Neuroform } 2\end{array}$ & 3 \\
\hline 2 & $\begin{array}{l}\text { L M2/M3 bifurcation } \\
L \text { M2 }\end{array}$ & ReoPro, Activase & Intra, Post & Maverick, $1.5 \times 9 \mathrm{~mm}$ & 2 \\
\hline 3 & $\begin{array}{l}\text { L M3 } \\
\text { L M2/M3 bifurcation }\end{array}$ & ReoPro & Post & Maverick, $2.5 \times 15$ mm, Wingspan, Enterprise & 2 \\
\hline 4 & $\begin{array}{l}\text { L M3 } \\
\text { L M2 } \\
\text { L M1 }\end{array}$ & ReoPro & Pre & Maverick, $2.0 \times 15 \mathrm{~mm}$ & 4 \\
\hline 5 & $\begin{array}{l}\text { L M2 } \\
\text { L M1 }\end{array}$ & ReoPro & Post & $\begin{array}{l}\text { Merci } \\
\text { Maverick, } 1.5 \times 20 \mathrm{~mm}\end{array}$ & 2 \\
\hline 6 & $\begin{array}{l}\text { R M3 } \\
\text { R M2 }\end{array}$ & Alteplase & Pre, Intra & Maverick, $1.5 \times 9 \mathrm{~mm}$ & 4 \\
\hline 7 & R M3 & IV rtPA, ${ }^{a}$ ReoPro, nitroglycerin & Post & Maverick, $1.5 \times 15 \mathrm{~mm}$ & 3 \\
\hline
\end{tabular}

\begin{tabular}{|c|c|c|c|c|c|c|c|}
\hline $\begin{array}{l}\text { Case } \\
\text { No. }\end{array}$ & Age $(y r) / S e x$ & Occlusion Sites & Stent & $\begin{array}{l}\text { TIMI } \\
\text { Grade }\end{array}$ & $\begin{array}{l}\text { Presenting } \\
\text { NIHSS }\end{array}$ & $\begin{array}{l}\text { Discharge } \\
\text { NIHSS }\end{array}$ & $\begin{array}{l}\mathrm{mRS} \\
\text { Score }\end{array}$ \\
\hline 1 & $28 / \mathrm{M}$ & L M2 & Yes & 3 & 8 & 2 & 1 \\
\hline 2 & $76 / F$ & $\begin{array}{l}\text { L M2/M3 bifurcation } \\
\text { L M2 }\end{array}$ & No & 4 & 18 & 0 & 0 \\
\hline 3 & $60 / \mathrm{M}$ & $\begin{array}{l}\text { L M3 } \\
\text { L M2/M3 bifurcation }\end{array}$ & Yes & 4 & 9 & 1 & 1 \\
\hline 4 & $74 / F$ & $\begin{array}{l}\text { L M3 } \\
\text { L M2 } \\
\text { L M1 }\end{array}$ & No & 4 & 9 & 0 & 0 \\
\hline 5 & $77 / F$ & $\begin{array}{l}\text { L M2 } \\
\text { L M1 }\end{array}$ & No & 3 & 20 & 4 & 0 \\
\hline 6 & $80 / \mathrm{M}$ & $\begin{array}{l}\text { R M3 } \\
\text { R M2 }\end{array}$ & No & 4 & 18 & $-^{\mathrm{a}}$ & 6 \\
\hline 7 & 76/M & R M3 & No & 3 & 15 & 0 & 0 \\
\hline
\end{tabular}

a Patient died after clinical deterioration following initial treatment.

80 years). Four patients were men and 3 were women. The mean NIHSS score at time of presentation was 14 (range, 8-20). Sites of occlusion were in M2 for 5 patients, at the M2/M3 bifurcation for 2 patients, and in the M3 in 4 patients. Two patients received concomitant treatment for an M1 occlusion. The mean time elapsed from onset of symptoms to commencement of treatment was 5 hours (range, 2.4-13 hours). One patient fell well outside the window for both IV and IA rtPA, but the decision was made to attempt endovascular treatment.

\section{Patient Selection}

A chart review was performed with institutional review board approval from the AMC. This was a retrospective chart review of patients with total or near-total occlusion of the distal MCA. All PTA cases from September 2005 and December 2007 were reviewed, and those involving distal MCA occlusions were included in the study. For the purpose of our study, M1 refers to the horizontal branch of the MCA; M2 includes the superior and inferior divisions in relation to the Sylvian fissure branching from M1. The M2/M3 bifurcation refers to branching of the M3 vessels off the M2 at the level of the Sylvian fissure. Distal MCA occlusion includes any occlusion within the M2 or M3 branch. In 2 patients, treatment was concomitantly performed for occlusion within the M1 segment of the MCA. Of the 9 patients who underwent distal PTA, we excluded 2 who presented with ruptured aneurysms and had distal MCA occlusions during endovascular procedures, leaving a total of 7 patients. Five patients presented to the AMC ED with strokelike symptoms and focal neurologic deficits. The remaining 2 patients developed strokelike symptoms during an inpatient stay for an unrelated illness.

\section{Initial Presentation}

Patients presenting with acute strokelike symptoms either to the ED or during inpatient stay were evaluated with a noncontrast CT and CTA of the head. Diagnosis of arterial occlusion was then confirmed with cerebral angiography.

Patients' symptoms were graded by an attending neurologist and assigned a score on the NIHSS. Patients were then referred for immediate treatment by an endovascular neurosurgeon.

\section{Endovascular Procedure}

Consent for diagnostic angiography with intent to treat was obtained by the patient or patient's proxy before the procedure. Patients were then placed under general anesthesia. They were properly anticoagulated with heparin, and percutaneous femoral artery access was achieved with a $6 \mathrm{~F}$ sheath. A diagnostic angiogram of the cerebral vasculature was obtained from the common carotid artery or ICA. After identifying the site of occlusion in the MCA, we used a microcatheter and microwire system to cross the occluded vessel. Once angiography confirmed that the microcatheter remained within the vessel distal to the occlusion, a Transcend Floppy (Boston Scientific, 

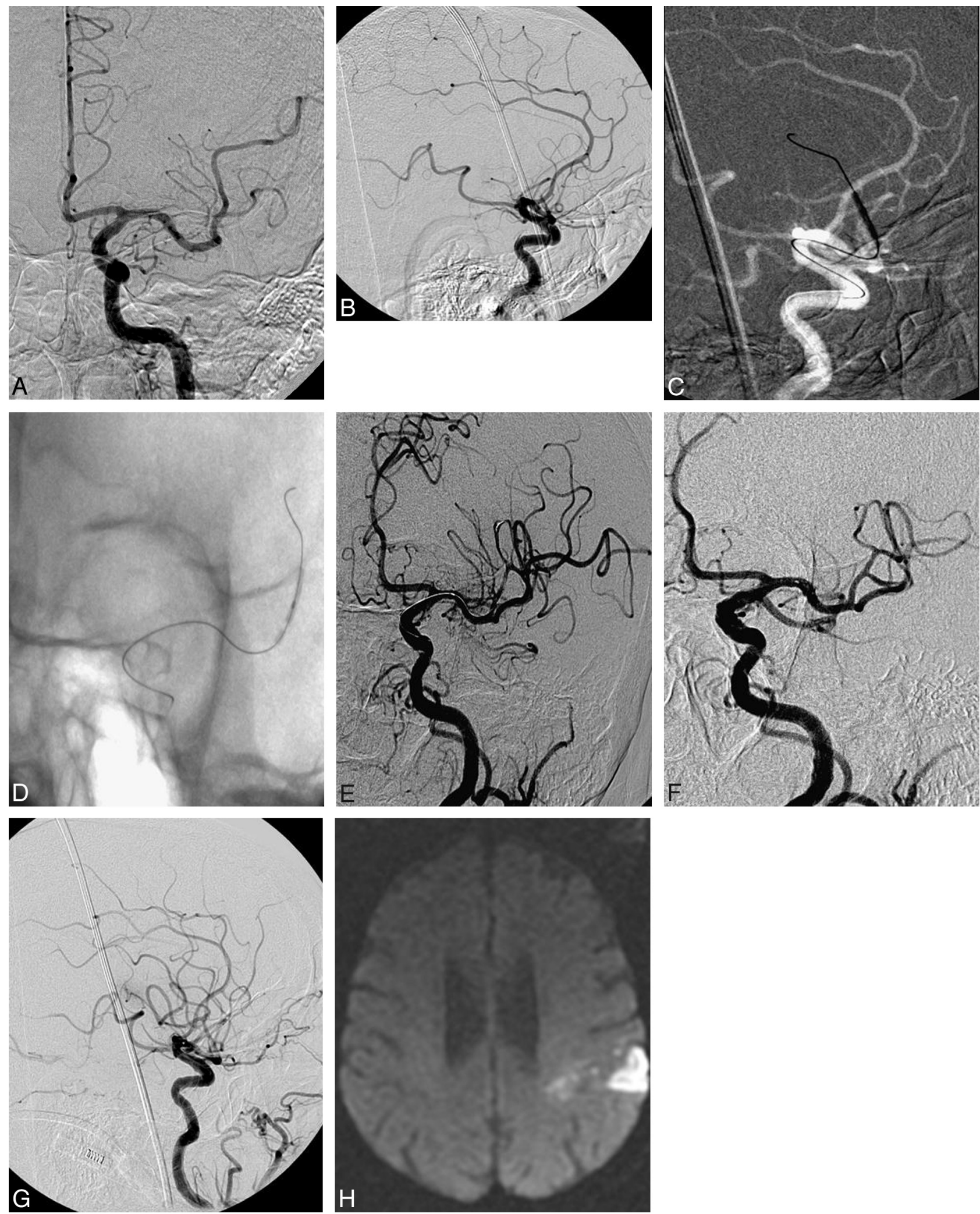

Fig 1. $A$ and $B$, A 76-year-old RHF 4.5 hours after onset of R hemiparesis, with global aphasia (NIHSS 18). CTA and CT perfusion show increased mean transit time in the L MCA region and M2 occlusion. Anteroposterior (AP) projection and lateral $(B)$ views demonstrate near occlusion of the M2 branch (measuring $1.2 \mathrm{~mm}$ ). $C$ and $D$, Inflated balloons of $1.5 \times 15 \mathrm{~mm}$. $E$, Oblique view after the first round of PTA and thrombolytics demonstrates incomplete recanalization with distal flow restoration. $F$ and $G, A P(F)$ and lateral $(G)$ views postthrombolytics and PTA in the late arterial phase $(G)$. H, Diffusion-weighted imaging section shows the restricted region postprocedure.

Natick, Massachusetts) or an Asahi Prowater (Abbott Vascular Devices, Redwood City, California) exchange-length microguidewire was inserted coaxially through the microcatheter.
A Maverick angioplasty balloon (Boston Scientific) was advanced over the exchange-length microguidewire to the occlusion site, and angioplasty was performed with a total inflation-deflation time of 

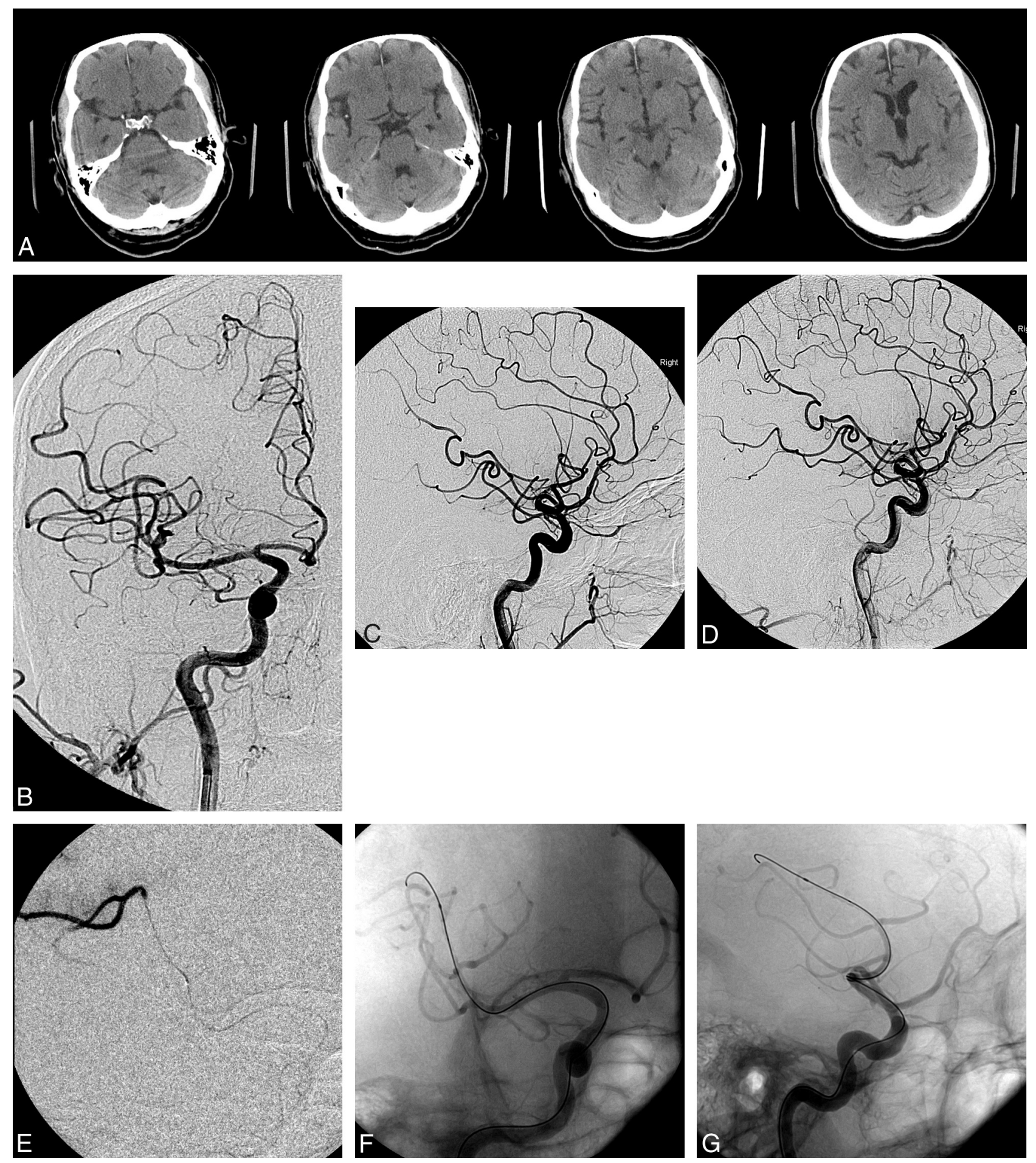

Fig 2. A, Preoperative CT scans show normal CT findings with no early ischemic changes. On the second image from the left, there is hyperattenuation in the right Sylvian fissure consistent with a thrombosed $\mathrm{M} 2$ or $\mathrm{M} 3$ branch. $B$ and $C, \mathrm{AP}(B)$ and lateral $(C)$ preoperative angiograms. Note a single MCA branch occlusion affecting the pre- and postcentral gyrus region in the posterior frontal lobe. $D$, Lateral view of the vessel occlusion. $E$, AP oblique view with a microcatheter injection demonstrating patency of the larger branch beyond the vessel occlusion. Note the distal embolus. Fand G, AP oblique $(F)$ and lateral $(G)$ views. Native images demonstrate distal catheterization of the MCA branch with an angioplasty balloon and wire. $H$ and I, Angioplasty balloon inflated in the M3 segment of the MCA. Angiogram demonstrates partial recanalization of the MCA branch. $J$ and $K$, Final AP $(J)$ and lateral $(K)$ images demonstrate hyperemia on the lateral view of the recanalized portion of the posterior frontal region. Branch occlusion is still present on the lateral view but with a smaller area of decreased perfusion. L, Postoperative head CT scans with normal findings show no infarct.

5 minutes per angioplasty. The size ranges of Maverick balloons used in the procedure included diameters of $1.5-2.5 \mathrm{~mm}$ and lengths of 9-20 mm (Table 1). After flow had been re-established, antispasmolytics (nitroglycerin or verapamil) and/or antithrombolytic agents (including IA rtPA and/or abciximab [ReoPro]) were delivered via microcatheter to prevent postprocedural vasospasm or re-thrombosis. Before device removal, a final angiogram of the cerebral circulation was obtained to demonstrate re-establishment of flow in the affected territory. Treatment materials were removed, and femoral artery hemostasis was achieved with an Angio-Seal closure 

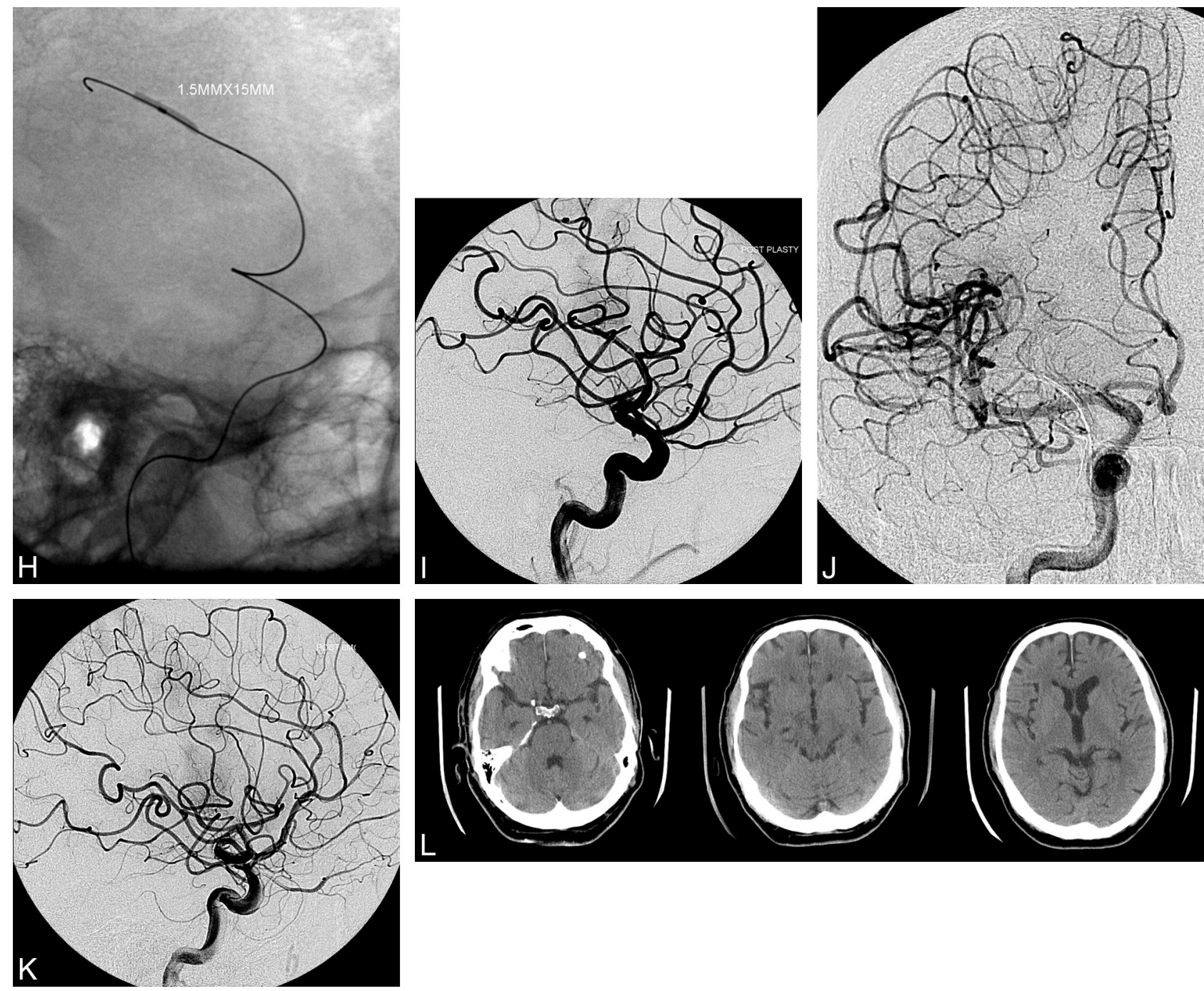

Fig. 2. Continued

device (St Jude Medical, Minnetonka, Minnesota) and manual pressure.

\section{Outcome Assessment}

Immediate recanalization was assessed by using the modified TIMI scale. ${ }^{10}$ This scale defines flow and perfusion as the following: grade 0 , no flow; grade 1, some penetration past the site of occlusion but no flow distal to the occlusion; grade 2, distal perfusion but delayed filling in all vessels; grade 3 , distal perfusion with adequate perfusion in less than half of the distal vessels; and grade 4, distal perfusion with adequate perfusion in more than half of the distal vessels. ${ }^{8}$ Vessel patency was reassessed with subsequent follow-up angiograms.

Clinical outcomes were immediately assessed with a postprocedural NIHSS by an endovascular neurosurgeon. An mRS was assigned to patients on the basis of their associated symptoms at their most recent follow-up appointment with an attending neurologist.

\section{Results}

\section{Treatment Results}

All patients underwent IA balloon angioplasty at the site of occlusion. One patient underwent angioplasty following failed treatment with the Merci retriever (Concentric Medical,
Mountain View, California) due to vessel tortuosity. One patient required angioplasty for distal MCA occlusion following successful Merci clot retrieval within the M1 branch. Chemical thrombolysis, spasmolysis, and anticoagulation were achieved with combinations of rtPA, nitroglycerin, and ReoPro (Table 1). Two patients underwent postangioplasty stent placement in the affected vessel (Table 1). Stent placement included the following: Wingspan stent (Boston Scientific) and Enterprise self-expanding stent (Cordis, Miami Lakes, Florida).

Vessel recanalization was assessed by using the modified TIMI scale. Complete flow (modified TIMI grade 4) was achieved in 4 patients, with recanalization with the remaining perfusion deficit (ie, distal smaller vessel occlusion) (modified TIMI grade 3 ) in 3 patients (Table 2). The mean time from onset of symptoms to successful recanalization of the vessel based on angiographic assessment was 6.5 hours (range, 4.5-15 hours). Treatment complications included 1 mortality, whereby the patient, despite having an excellent angiographic outcome with no signs of contrast extravasation, had hemorrhagic conversion of ischemic stroke 5 days postprocedure, from which he did not recover. No patients required additional procedures for their strokes. 


\section{Clinical Outcomes}

At time of discharge, the mean NIHSS was 1.2 (range, 0-4). One patient did not recover from his embolic event despite complete restoration of flow, and he died during his inpatient stay. The mean length of the hospital stay was 10 days (range, 5-30). The mean mRS at most recent follow-up was 0.3 (range, $0-1$ ), excluding the 1 mortality.

\section{Illustrative Cases}

Case 1. A 76-year-old right-handed woman (Patient 2) presented to the ED with acute right-sided hemiplegia, aphasia, and an NIHSS score of 18 by an attending neurologist. Hemorrhagic stroke was ruled out by noncontrast head CT, and CTA identified the etiology as an occlusion of the left M2 and M2/M3 bifurcation. The patient had been last seen symptom-free 4 hours 30 minutes before treatment, and her hemiplegia and aphasia had been present for at least 3 hours. Due to the patient's speech deficit, consent to treat was obtained by the patient's family, at which point she was taken to the angiography suite for definitive diagnosis and treatment.

The left ICA was catheterized under direct fluoroscopy through femoral access. Angiography confirmed an occlusion within the distal inferior M2 extending through the M2/M3 bifurcation into the proximal M3 segment (Fig 1). A $1.5 \times$ $9 \mathrm{~mm}$ Maverick angioplasty balloon was navigated over a guidewire within the proximal M3 to the main site of occlusion at the inferior M2. The balloon was inflated to $5 \mathrm{~atm}$ for 2 minutes, held for 1 minute, and then deflated for 2 minutes for a total of 5 minutes of angioplasty. IA thrombolytics (5-mg ReoPro and 3-mg rtPA) were delivered to the lesion, at which point control angiography showed good restoration of flow in the proximal M3, M2/M3 bifurcation, and M2 branches. However, due to some residual slow filling during the capillary phase (modified TIMI grade 3 ), an additional angioplasty of the M2 branch proximal to the M2/M3 bifurcation was performed, along with a final administration of $9 \mathrm{mg}$ of ReoPro and $3 \mathrm{mg}$ of rtPA before completion of the procedure. A final angiogram demonstrated excellent flow, with complete patency (modified TIMI grade 4) through the M2 and into the M2/M3 bifurcation with no evidence of contrast extravasation.

Within 24 hours of endovascular treatment, the patient's aphasia had resolved and her right-sided hemiplegia had improved dramatically. A follow-up MR imaging of the brain did demonstrate some left parietal signal-intensity abnormalities consistent with a small MCA embolic stroke. The patient continued to improve during her inpatient stay and was discharged on hospital day 9 on enoxaprin and warfarin (the patient had a history of atrial fibrillation). Her NIHSS score at discharge had resolved to a 0 . At 3 months' postprocedure, she presented without focal neurologic deficits ( $\mathrm{mRS} 0$ ).

Case 2. A 76-year-old man (Patient 7) presented to the ED at AMC with acute onset of left-sided upper and lower extremity weakness and expressive aphasia. On arrival at the ED, he underwent CT angiography, which was unable to identify an occlusive lesion but did show significant stenosis of the right proximal MCA and M2 branch. Because of the severity of his clinical symptoms, the patient received IV tPA at 2.5 hours from the onset of symptoms. The patient failed to improve following administration of IV rtPA, and after dis- cussion with the patient's family as well as the attending neurologist and endovascular neurosurgeon, consent was obtained and the patient was taken for angiography and possible intervention.

The patient was intubated and placed under general anesthesia. Angiography demonstrated occlusion within the M2 branch of the right MCA (Fig 2). A guidewire was used to navigate into the affected vessel. A $1.5 \times 15 \mathrm{~mm}$ Maverick balloon was exchanged over the guidewire, and angioplasty of the M2 lesion was performed in sequential fashion, for a total of 3 inflation/deflation cycles. The balloon was retracted into the proximal M1 branch, and contrast agent was injected demonstrating recanalization of the proximal M2 division. The balloon catheter was removed, and ReoPro and nitroglycerin were injected into the M2 segment from the microcatheter. A final angiogram demonstrated good filling of the M2, with capillary blush and without extravasation of contrast. A small filling defect of the M3 branch was noted; however, due to the length of the procedure and multiple previous angioplasties, the decision was made to refrain from further intervention.

The patient was transferred to the surgical intensive care unit, where he remained in stable condition and was extubated the following day without incident. He had return of left-sided strength, movement, and speech by postoperative day 1 ; aspirin and clopidogrel (Plavix) were initiated. The patient was discharged on hospital day 5 with an NIHSS score of 0 .

The patient's most recent follow-up was 10 months after the procedure, where he stated he was free from any motor, sensory, or speech dysfunction (mRS 0).

\section{Discussion}

Although IV rtPA can be sufficient for restoration of flow in acute cerebral ischemia within the 3-hour time window, $\leq 30 \%$ of occlusions within the M1 segment of the MCA do not respond to IV rtPA alone. ${ }^{1}$ In fact, $58 \%$ of treated patients still have significant disability after having been given IV rtPA in the landmark pivotal trial. ${ }^{1}$ As such, the concomitant use of mechanical devices to improve restoration rates is essential to improving patient outcomes.

In regard to our patients, after discussion between a team of neurologists and endovascular neurosurgeons, we thought that these distal MCA lesions warranted mechanical intervention as opposed to more conservative measures. The decision process involved not only the patient's angiographic appearance but also the clinical status and changes on CT perfusion scans. In addition, many of these patients had significant language difficulties correlating with their left MCA lesions, which required us to be somewhat more aggressive with their treatment.

Initial studies have sought to examine the benefits of combined IV rtPA with IA intervention within the 3-hour window. Sugiura et $\mathrm{al}^{6}$ compared outcomes with combined IV and IA rtPA, mechanical guidewire disruption, and PTA versus IV rtPA alone within the 3-hour window. Among a treatment group of 23 patients, they found that follow-up mRS scores were favorable (mRS 0 and 1 ) for $63 \%$ of patients in the combined group versus $15 \%$ of patients in the IV alone group. Although PTA was used in the combined group, use of PTA specifically in the distal MCA territory was not described by the study authors. 
The purpose of this study was to determine if PTA could be used to treat vessels traditionally thought of as being too small and tortuous for treatment other than microcatheterization and medication administration. This traditional belief may be due to the idea that the risk of inflating a balloon within the distal MCA was thought to be too great. Fortunately, with the use of more steerable microwires and microcatheters, we were able to perform PTA on the occluded vessel routinely.

The advent of small coronary balloons $(1.5 \mathrm{~mm}$ in diameter) has increased the distal limit of successful recanalization of intracerebral occlusions as well as decreased the risk of balloon-associated vessel damage. The balloons were highly navigable over a 0.014 -inch-diameter exchange-length microwire. Angioplasty was performed slowly as previously demonstrated by Connors and Wojak. ${ }^{11}$ The principle of slow inflation (as long as 7 minutes for complete inflation) and the smaller diameter of the deflated balloon in relation to the vessel were found to decrease the incidence of dissection and restenosis and improve clinical outcomes when compared with balloons with enlarged vessel diameters and rapid inflation times (15-30 seconds). ${ }^{11}$ In addition, slow inflation/ deflation times have allowed us to successfully deploy oversized stents in the canine basilar artery compared with a high rate of vessel rupture with rapid deployment of the stent. Successful and safe canalization of occluded regions of narrow and tortuous distal vasculature, along with a slow inflation time, is essential to achieving vessel patency while at the same time lowering the risk of distal clot emboli through lysis or peripheral compaction.

PTA has been used previously in ischemic stroke with moderate success. ${ }^{8,12}$ For fear of rupturing the intracranial vessel, balloons that are undersized to the parent vessel are often used. $^{11}$ In our experience, more commonly treated large-vessel occlusions within the cerebral vasculature, such as those within the M1, ICA bifurcation, and ICA terminus, often open with angioplasty but are left with a significant residual stenosis and are subsequently prone to reocclusion. Stent placement can improve this complication and obtain a more significant gain. ${ }^{4}$ Mechanical clot-retrieval devices also have moderate success at restoring patency of the proximal intracranial vessels (such as was the case with patient 6) but are too large to be used in small branches of the MCA (M2-M3), whose diameter is between 1 and $2 \mathrm{~mm}$, as is reinforced by the 1 case of attempted mechanical thrombectomy within our patient series. In our experience, distal angioplasty appears to be more successful angiographically than proximal larger vessel angioplasty. There is rarely a residual stenosis or limitation of flow at the initial site of occlusion. Our hypothesis is that the reference diameter is similar in size to the smallest coronary artery balloon $(1.5 \mathrm{~mm})$, so the occluded site is either broken apart, embolized, or compressed into the wall of the vessel, resulting in complete recanalization at the site of angioplasty.

In our study, patients who had significant neurological disabilities and small vessel occlusions underwent angioplasty as the primary therapy with reversal of deficits in all but 1 patient. In addition, 2 patients with significant neurological disability underwent treatment of a proximal M1 occlusion and with recanalization a subsequent distal lesion was discovered and treated primarily with angioplasty with good success and reversal of the majority of their symptoms.
Other mechanical alternatives to PTA in the distal vasculature include self-expanding stents and deflated-balloon disruption. Levy et $\mathrm{al}^{4}$ used self-expanding stents along with IA thrombolysis for 19 patients, 7 of whom were treated in the MCA territory (M1 or M2). Risks associated with this procedure included hemorrhage without adverse clinical effects in 1 patient. The recanalization rate using this technique was $79 \%$ overall and $85 \%$ in the MCA territory. One potential drawback to this technique is that stent placement requires postprocedural antiplatelet administration. These patients need to be on either 2 oral antiplatelet agents or an IV IIb/IIIa glycoprotein receptor antagonist, which may lead to a higher risk of postprocedural intracranial hemorrhage. With PTA, there may still be this associated risk; however, dual antiplatelet medications may not be as essential.

Ikushima et $\mathrm{al}^{7}$ presented a study whereby recanalization to the proximal and distal MCA was achieved by using a deflated balloon over a guidewire to disrupt the clot as opposed to conventional PTA. This study demonstrated good restoration of flow in 7 patients. However, mechanical disruption of the clot may increase the risk of distal emboli. While this occurred only once in our series, it is something to be cognizant of with microcatheterization of clot. One other advantage of complete PTA over clot disruption is the potential decrease in treatment time. Immediate restoration of flow with luminal balloon dilation may have some advantages over clot disruption with a deflated balloon, which may take repeated passes with the device to restore flow.

Although radiographic outcomes do not necessarily mirror treatment success in acute cerebral ischemia, revascularization of the affected vessel should be the primary goal of the treating neuroendovascular specialist when performing IA therapy within the 6-hour window. We were able to show, in our study, that PTA successfully restored flow (modified TIMI grade $3-4$ ) in $100 \%$ of our patients. Furthermore, the use of mechanical angioplasty in distal vessels may increase total flow and decrease time to recanalization as opposed to other therapies discussed.

The distal location of the occlusion may also extend the potential therapeutic time window as the chance of pial collaterals preventing completed infarction within 6 hours increases. These patients with distal location likely have decreased blood flow with resultant ischemic penumbra that is reversible with restoration of flow. One important caveat of this is that these patients' natural histories may also be better than those of patients with M1 or ICA occlusions. These patients, though presenting with high NIHSS and significant disability, may make significant recovery without reperfusion therapy. Overall, the study was promising and makes the treatment of smaller intracranial vessels possible and potentially clinically rewarding.

There are a few limitations to our study: 1) the relatively small population of 7 patients, 2) the retrospective nature of the study, and 3 ) the persistent risk of perforation and subsequent hemorrhage with mechanical intervention. In addition, in the 2 patients who had their M1 occlusion treated before their distal MCA lesion was found, it is difficult to determine the significance of treating the distal lesions. At the same time, both patients did exceptionally well postintervention, and we make the assumption that treating the distal MCA lesions 
must have contributed, at least in part, to this improvement, but to what degree is difficult to say. While there are clearly limitations to generalizing this small retrospective study, it does suggest a potential role of endovascular therapy for a subset of patients with acute occlusion within the distal MCA. Further studies with increased sample size and prospective analysis will hopefully confirm this.

\section{Conclusions}

This case series demonstrates that PTA of the distal MCA with a microballoon is a safe and effective treatment for acute ischemic stroke and can dramatically reverse neurologic deficits with minimal complications. While this case series shows great potential for PTA, a larger prospective study would be of benefit to better delineate the parameters for its use.

\section{References}

1. Tissue plasminogen activator for acute ischemic stroke: The National Institute of Neurological Disorders and Stroke rt-PA Stroke Study Group. N Engl J Med 1995;333:1581-87

2. Furlan A, Higashida R, Wechsler L, et al. Intra-arterial prourokinase for acute ischemic stroke: the PROACT II study - a randomized controlled trial. Prolyse in Acute Cerebral Thromboembolism. JAMA 1999;282:2003-11
3. Smith WS, Sung G, Saver J, et al. Mechanical thrombectomy for acute ischemic stroke: final results of the Multi MERCI trial. Stroke 2008;39:1205-12

4. Levy EI, Ecker RD, Horowitz MB, et al. Stent-assisted intracranial recanalization for acute stroke: early results. Neurosurgery 2006;58:458-63

5. Sauvageau E, Levy EI. Self-expanding stent-assisted middle cerebral artery recanalization: technical note. Neuroradiology 2006;48:405-08

6. Sugiura S, Iwaisako K, Toyota S, et al. Simultaneous treatment with intravenous recombinant tissue plasminogen activator and endovascular therapy for acute ischemic stroke within 3 hours of onset. AJNR Am J Neuroradiol 2008;29:1061-66

7. Ikushima I, Ohta H, Hirai T, et al. Balloon catheter disruption of middle cerebral artery thrombus in conjunction with thrombolysis for the treatment of acute middle cerebral artery embolism. AJNR Am J Neuroradiol 2007; 28:513-17

8. Ringer AJ, Qureshi AI, Fessler RD, et al. Angioplasty of intracranial occlusion resistant to thrombolysis in acute ischemic stroke. Neurosurgery. 2001;48: 1282-88, discussion $1288-90$

9. Von Kummer R, Hacke W. Safety and efficacy of intravenous tissue plasminogen activator and heparin in acute middle cerebral artery stroke. Stroke 1992;23:646-52

10. Qureshi AI, Ali Z, Suri MF, et al. Intra-arterial third-generation recombinant tissue plasminogen activator (reteplase) for acute ischemic stroke. Neurosurgery 2001;49:41-48, discussion 48-50

11. Connors JJ 3rd, Wojak JC. Percutaneous transluminal angioplasty for intracranial atherosclerotic lesions: evolution of technique and short-term results. J Neurosurg 1999;91:415-23

12. Boulos AS, Levy EI, Bendok BR, et al. Evolution of neuroendovascular intervention: a review of advancement in device technology. Neurosurgery 2004;54:438-52, discussion 452-53 